Supporting Information

\title{
High-Detectivity Non-Fullerene Organic Photodetectors Enabled by a Cross-Linkable Electron Blocking Layer
}

Zhiming Zhong, ${ }^{\dagger}{ }^{\dagger}$ Feng Peng, ${ }^{\dagger}{ }^{\dagger} \S$ Zhenqiang Huang, ${ }^{\dagger}$ Lei Ying, $,{ }^{\dagger},, *$ Gang Yu, ${ }^{\dagger}$ Fei Huang, ${ }^{\dagger}, * *$ Yong $\mathrm{Cao}^{\dagger}$

$\dagger$ Institute of Polymer Optoelectronic Materials and Devices, State Key Laboratory of Luminescent Materials and Devices, South China University of Technology, Guangzhou 510640, China

§ South China Institute of Collaborative Innovation, Dongguan 523808, China

Corresponding Authors

*E-mail: msleiying@scut.edu.cn (L. Ying)

*E-mail: msfhuang@scut.edu.cn (F. Huang) 


\section{Contents}

1. Synthesis routines of XP1 and XP2

2. Synthesis of Donor Polymers

3. Solvent Rinsing Test

4. Cyclic Voltammetry Measurement

5. AFM Images

6. EQE Spectra of Devices with Different Interfacial Layers

7. Optimization of XP2

8. Device with Inverted Structure

9. Mott-Schottky Analysis

10. Performance of Peer OPDs 


\section{Synthesis of XP1 and XP2}
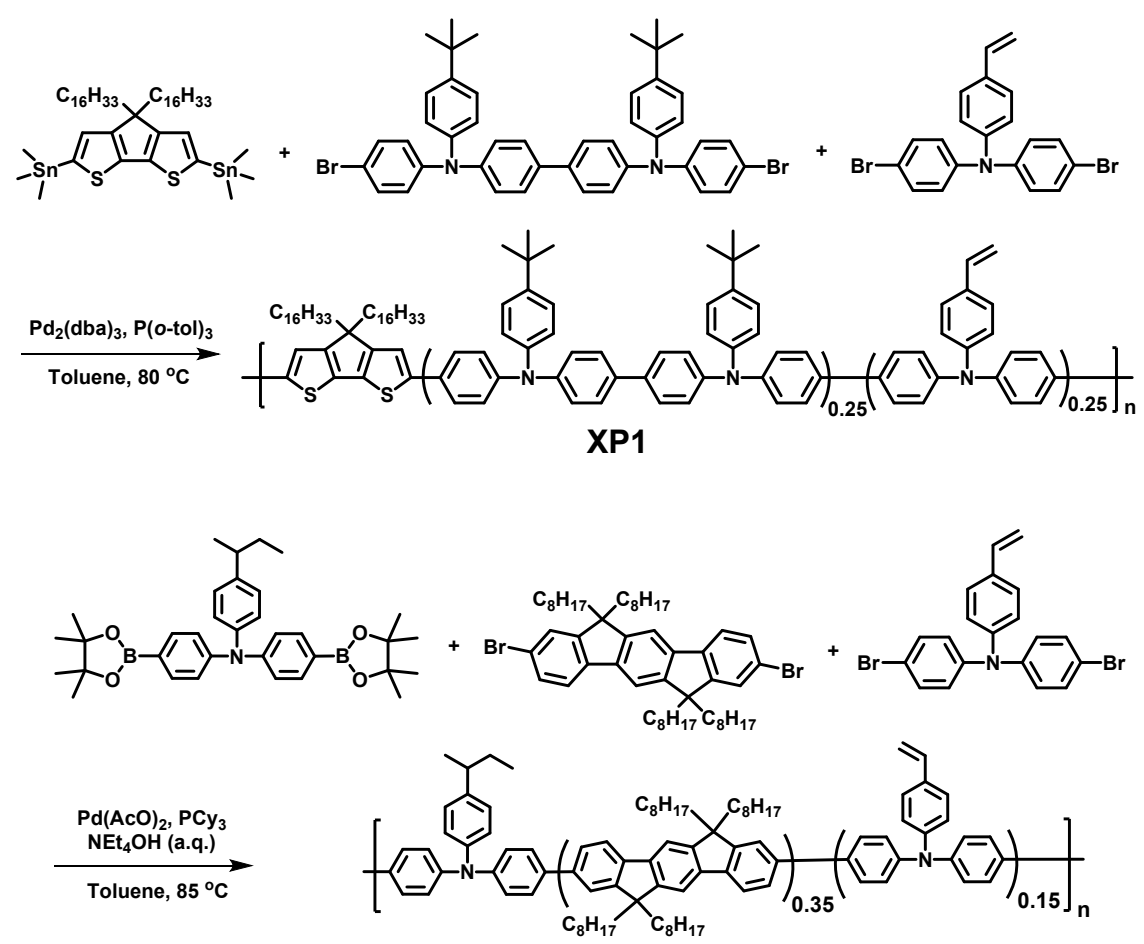

XP2

Scheme S1. The synthesis routines of XP1 and XP2.

\section{Materials}

(4,4-Dihexadecyl-4H-cyclopenta[2,1-b:3,4-b’']dithiophene-2,6-diyl)bis(trimethyl stannane) (M1), $\quad N^{4}, N^{4}$-bis(4-bromophenyl)- $N^{4}, N^{4}$-bis(4-(tert-butyl)phenyl)-[1,1'biphenyl]-4,4'-diamine (M2), 4-bromo- $N$-(4-bromophenyl)- $N$-(4-vinylphenyl)aniline (M3), 4-(sec-butyl)- $N, N$-bis(4-(4,4,5,5-tetramethyl-1,3,2-dioxaborolan-2-yl)phenyl) aniline (M4), 2,7-bis(4,4,5,5-tetramethyl-1,3,2-dioxaborolan-2-yl)-9,9-dioctylfluorene (M5) and 2,8-dibromo-6,6,12,12-tetraoctyl-6,12-dihydroindeno[1,2-b]fluorene (M6), were purchased from Dongguan Alda New Materials Technology Co., Ltd, and used as received without further purification.

\section{Synthesis of Cross-Linkable Polymers}

Polymer XP1 was synthesized via Suzuki polycondensation. Under an argon atmosphere, M1 (285.8 mg, $0.3 \mathrm{mmol}), \mathrm{M} 2$ (113.8 mg, $0.15 \mathrm{mmol}), \mathrm{M} 3$ (64.4 mg, 0.15 
mmol), tris(dibenzylideneacetone)dipalladium $(0) \quad(1.5 \quad \mathrm{mg}) \quad$ and $\operatorname{tris}(2-$ methylphenyl)phosphine ( $3 \mathrm{mg}$ ) were added into $6 \mathrm{~mL}$ toluene and heated to $80{ }^{\circ} \mathrm{C}$ for $24 \mathrm{~h}$. After being end-capped by tributyl(2-thiophenyl)tin $(30 \mathrm{mg})$ and 2bromothiophene $(50 \mathrm{mg})$, the reaction mixture was poured into $300 \mathrm{~mL}$ methanol. The crude polymer was washed with methanol and hexane in a Soxhlet apparatus and then dry off at $50{ }^{\circ} \mathrm{C}$ under vacuum to afford XP3 as a red floccule. ${ }^{1} \mathrm{H}$ NMR (400 MHz, $\left.\mathrm{CDCl}_{3}\right) \delta(\mathrm{ppm}):$ 7.65-7.40 (m, ArH), 7.38-7.25 (m, ArH), 7.21-6.95 (m, ArH), 6.75$6.64(\mathrm{~m}, \mathrm{CH}), 5.69(\mathrm{~d}, \mathrm{CH}), 5.20(\mathrm{~d}, \mathrm{CH}), 1.87\left(\mathrm{br}, \mathrm{CH}_{2}\right), 1.33\left(\mathrm{~s}, \mathrm{CH}_{3}\right), 1.30-1.10(\mathrm{~m}$, $\left.\mathrm{CH}_{2}\right), 1.02\left(\mathrm{br}, \mathrm{CH}_{2}\right), 0.86\left(\mathrm{t}, \mathrm{CH}_{3}\right)$. The number-average molecular weight $\left(M_{\mathrm{n}}\right)$ estimated by gel permeation chromatography (GPC) with tetrahydrofuran (THF) as eluent is $20.5 \mathrm{KDa}$, with corresponding polydispersity index of 2.1 .

Polymer XP2 was synthesized via Suzuki polycondensation under similar conditions as reference [S1]. Under an argon atmosphere, M4 (192.8 mg, $0.3 \mathrm{mmol})$, M5 (180.8 mg, $0.21 \mathrm{mmol}), \mathrm{M} 3(38.7 \mathrm{mg}, 0.09 \mathrm{mmol})$, palladium acetate $(2 \mathrm{mg})$ and tricyclohexylphosphine $\left(\mathrm{PCy}_{3}\right)(4 \mathrm{mg})$ were dissolved in $8 \mathrm{~mL}$ toluene and $1 \mathrm{~mL}$ tetraethyl ammonium hydroxide aqueous solution. The mixture was stirred and heated to $85{ }^{\circ} \mathrm{C}$ for $24 \mathrm{~h}$. Phenylboronic acid $(30 \mathrm{mg})$ and bromobenzene $(0.2 \mathrm{~mL})$ were successively added into the reaction mixture and respectively reacted for $6 \mathrm{~h}$ to terminate the end groups. After cooling to room temperature, the reaction solution was poured into $300 \mathrm{~mL}$ methanol. The precipitate was filtered and washed with methanol and hexane in a Soxhlet apparatus. The residue was redissolved in $20 \mathrm{~mL}$ chloroform and then reprecipitated in $300 \mathrm{~mL}$ methanol. The anticipated XP2 was obtained by filtration and drying at $50{ }^{\circ} \mathrm{C}$ under vacuum as a light yellow floccule. ${ }^{1} \mathrm{H}$ NMR (500 $\mathrm{MHz}, \mathrm{CD}_{2} \mathrm{Cl}_{2}$ ) $\delta$ 7.79-7.76 (br, ArH), 7.69-7.57 (br, ArH), 7.46-7.42 (br, ArH), 7.207.11 (br, ArH), 7.05-6.90 (br, ArH), 6.72-6.63 (br, CH), 5.62-5.60 (br, CH), 5.19-5.12 (br, $\mathrm{CH}$ ), 2.68-2.59 (br, CH), 2.10-1.93 (br, $\mathrm{CH}_{3}$ ), 1.62-1.58 (br, $\mathrm{CH}_{2}$ ), 1.26-1.00 (br, $\left.\mathrm{CH}_{2}\right), 0.86-0.72\left(\mathrm{br}, \mathrm{CH}_{2}\right), 0.69\left(\mathrm{br}, \mathrm{CH}_{3}\right)$. The $M_{\mathrm{n}}$ estimated by GPC with THF as the eluent is $46.8 \mathrm{KDa}$, with corresponding polydispersity index of 2.1 . 
(a)

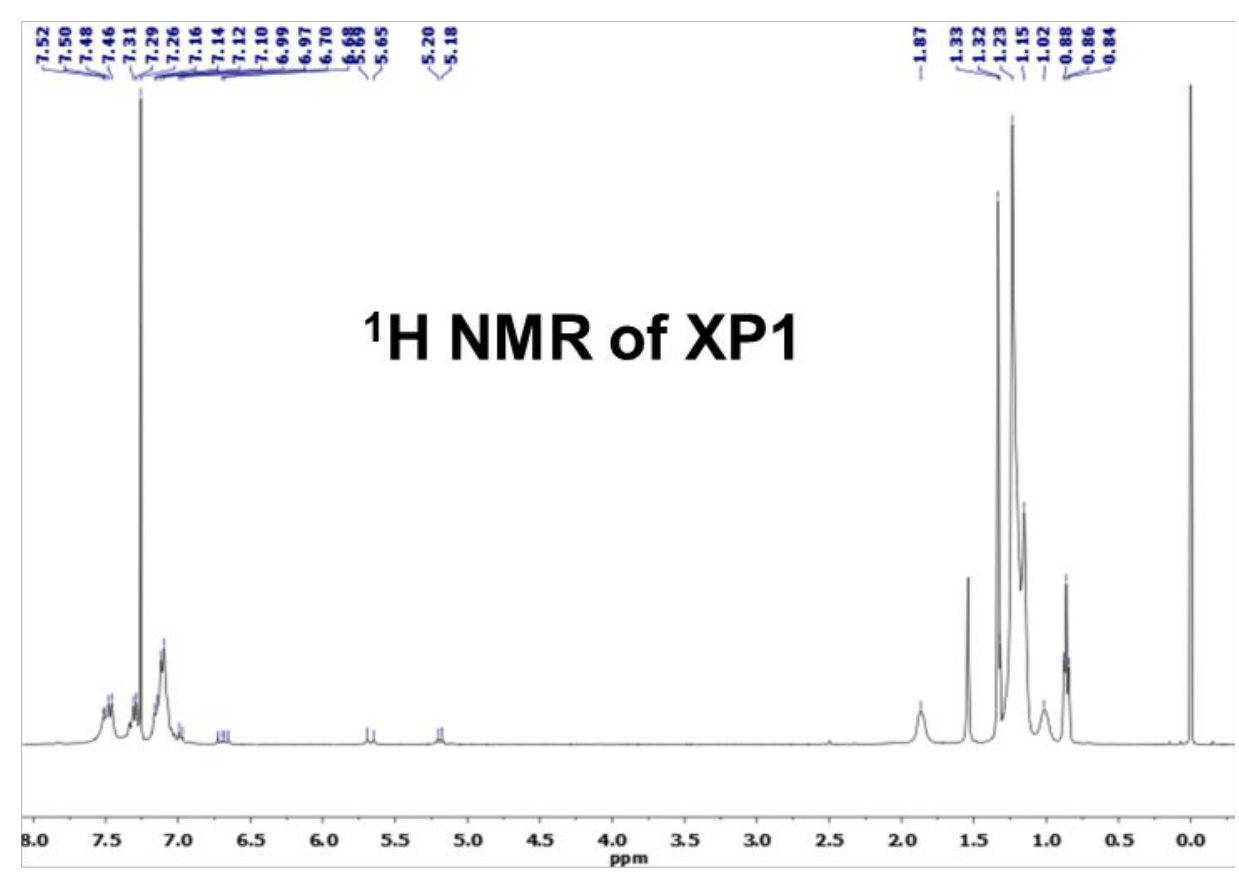

(b)

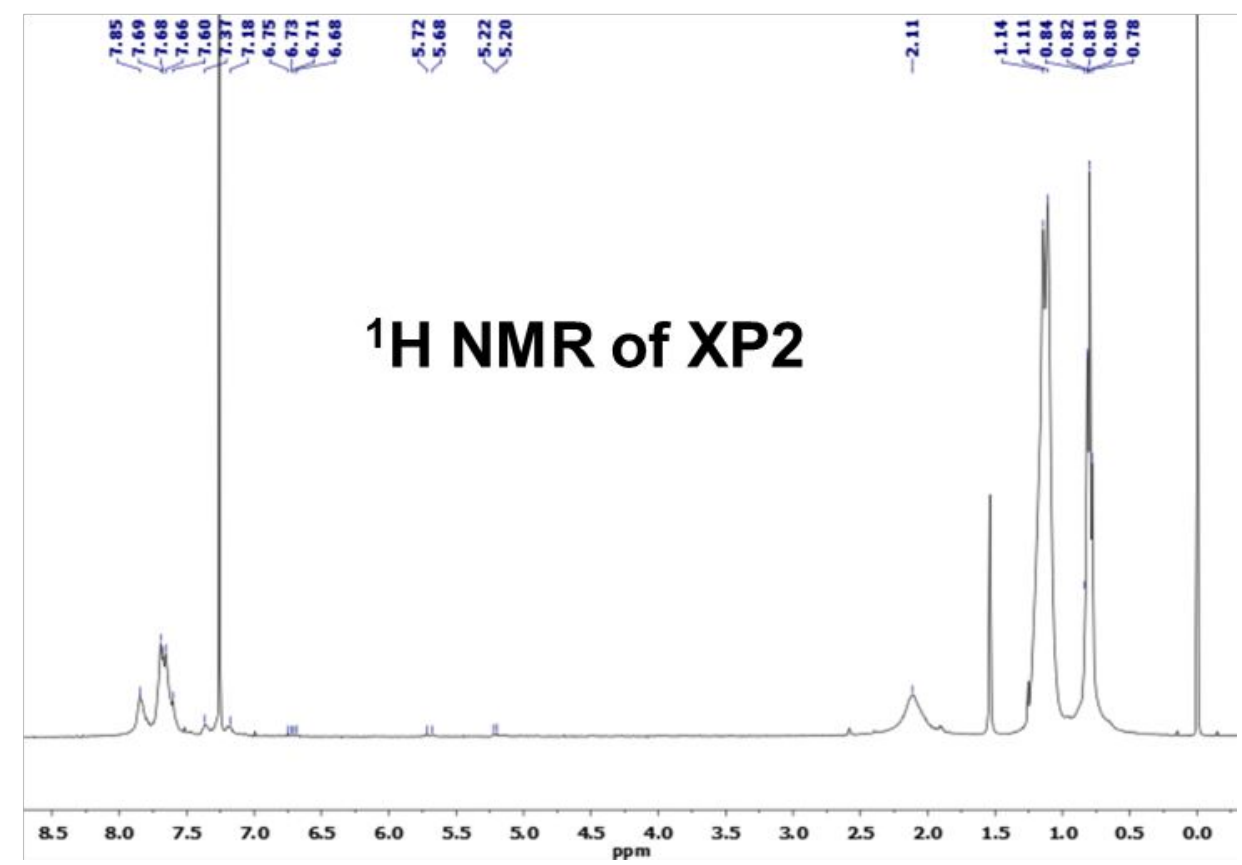

Figure S1. The ${ }^{1} \mathrm{H}$ NMR spectra of (a) XP1 and (b) XP2. 


\section{Synthesis of Donor Polymers}
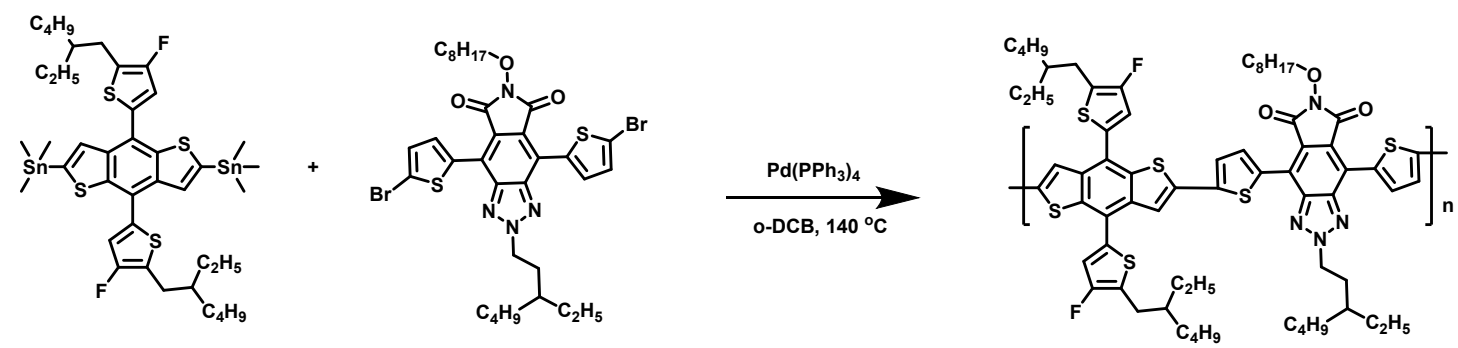

Scheme S2. The synthesis routines of PFT-OEHp. The synthesis of alkoxylated imidefunctionalized benzotriazole unit was according to the reference [S1, S2], with minor change in alkyl chain. 
3. Solvent Rinsing Test
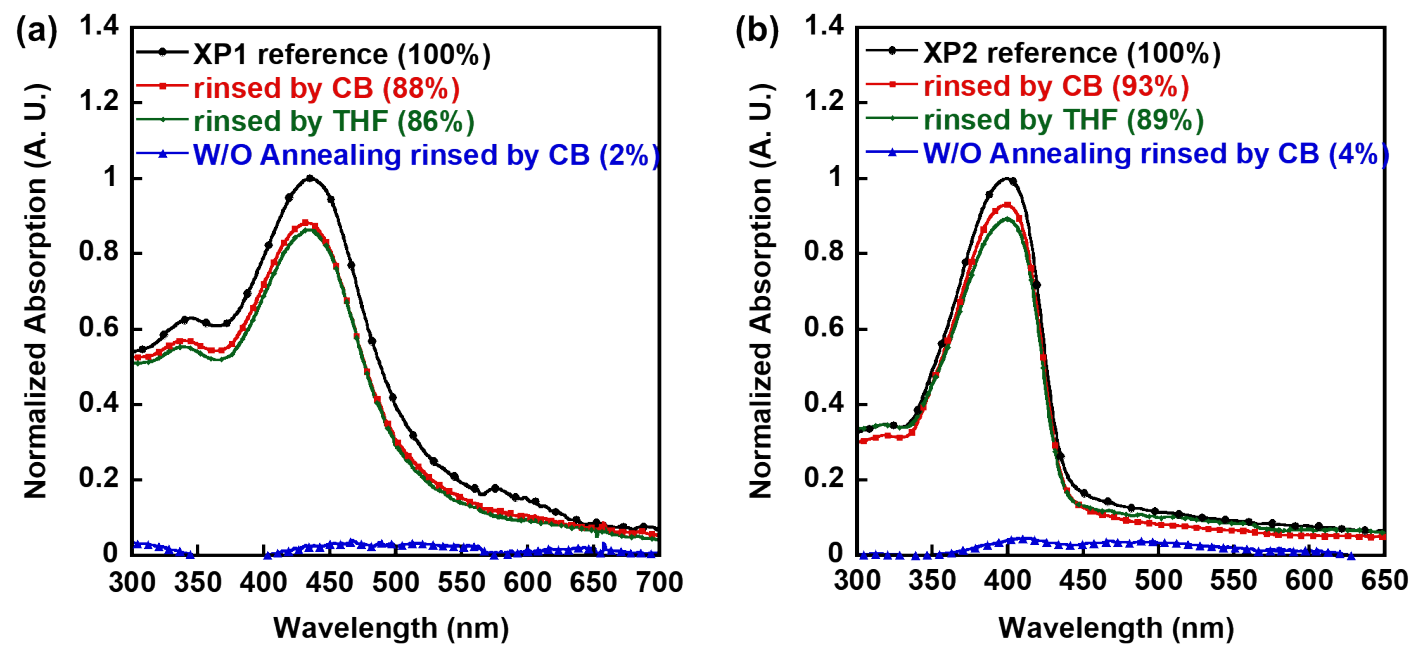

Figure S2. The normalized UV-vis absorption spectra of (a) XP1 and (b) XP2 before and after rinsing with chlorobenzene (CB) and tetrahydrofuran (THF). 


\section{Cyclic Voltammetry Measurement}

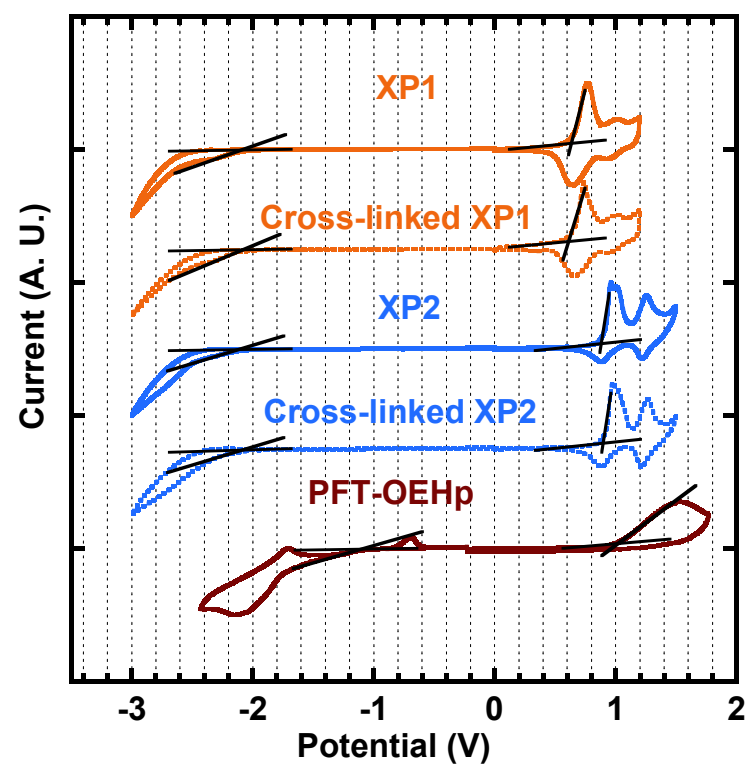

Figure S3. The cyclic voltammetry curves of XP1, XP2, cross-linked XP1, cross-linked XP2 and PFT-OEHp. Cyclic voltammetry was measured by using a CHI660A electrochemical workstation. The scan rate was $50 \mathrm{mV} \mathrm{s}^{-1}$ at room temperature under the inert atmosphere of argon. Tetra-(n-butyl)ammonium hexa-fluorophosphate ( $n$ $\left.\mathrm{Bu}_{4} \mathrm{NPF}_{6}, 0.1 \mathrm{M}\right)$ in acetonitrile solution was chosen as the electrolyte. 
Table S1. Electrochemical and optical properties of XP1, XP2 and PFT-OEHp.

\begin{tabular}{llll}
\hline & $E_{\text {HOMO }}$ & \multicolumn{1}{l}{$E_{\text {LUMO }}$} & $E_{\mathrm{g}}{ }^{\text {opt }}$ \\
& $(\mathrm{eV})^{a}$ & $(\mathrm{eV})^{a / b}$ & $(\mathrm{eV})^{c}$ \\
\hline XP1 & -5.04 & $-2.29 /-2.70$ & 2.34 \\
Cross-linked XP1 & -5.03 & $-2.30 /-2.70$ & 2.34 \\
XP2 & -5.30 & $-2.29 /-2.55$ & 2.75 \\
Cross-linked XP2 & -5.32 & $-2.30 /-2.55$ & 2.75 \\
PFT-OEHp & -5.38 & $-3.26 /-3.60$ & 1.78 \\
\hline
\end{tabular}

${ }^{a}$ derived from cyclic voltammetry

${ }^{b}$ derived from $E_{\mathrm{HOMO}}+E_{\mathrm{g}}{ }^{\text {opt }}$

${ }^{c}$ derived from absorption edge 


\section{AFM Images}
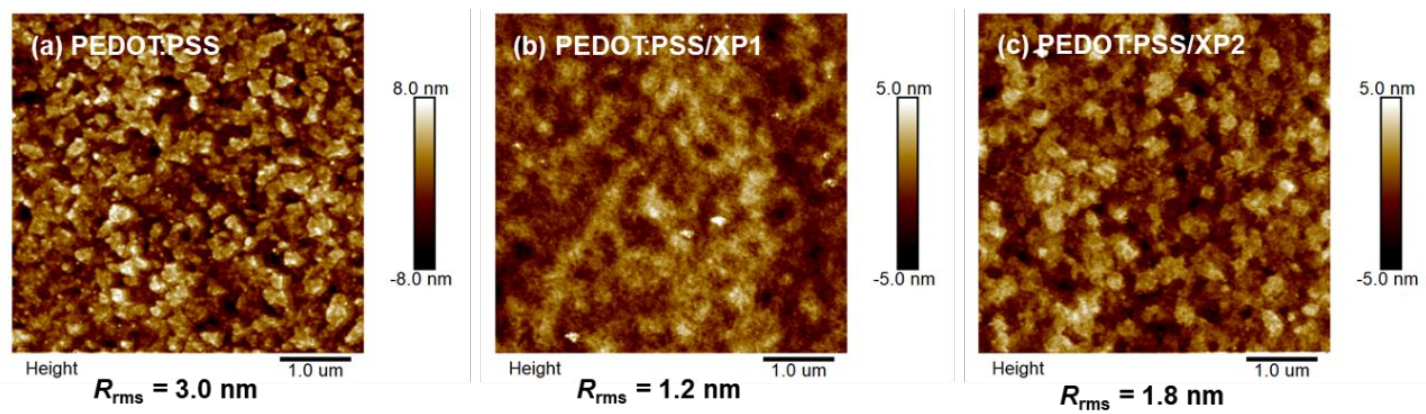

Figure S4. The surface height image of (a) ITO/PEDOT:PSS, (b) ITO/PEDOT:PSS/Cross-linked XP1 and (c) ITO/PEDOT:PSS/ Cross-linked XP2 scanned by atomic force microscopy. 
6. EQE Spectra of Devices with Different Interfacial Layers

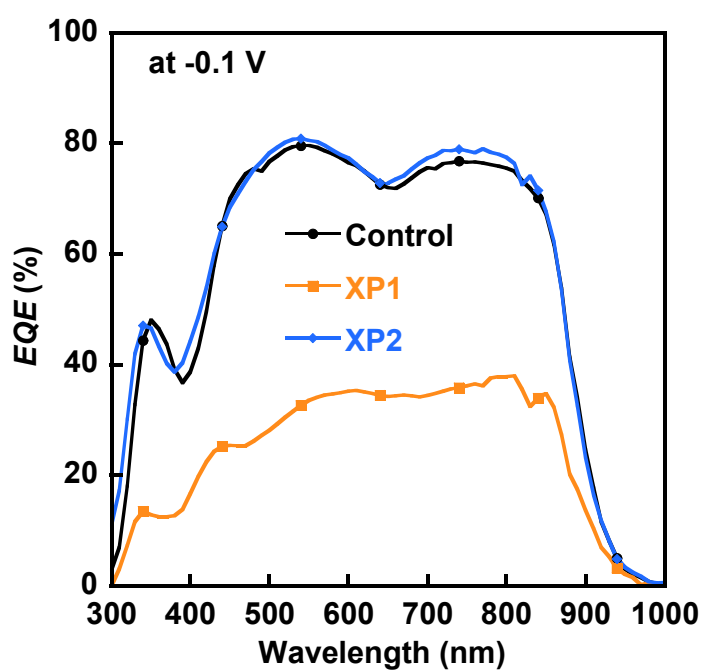

Figure S5. EQE spectra of PFT-OEHp:Y6 = 1:1.2 wt:wt devices at bias of $-0.1 \mathrm{~V}$ with and without a cross-linked interlayer. 


\section{Optimization of XP2}
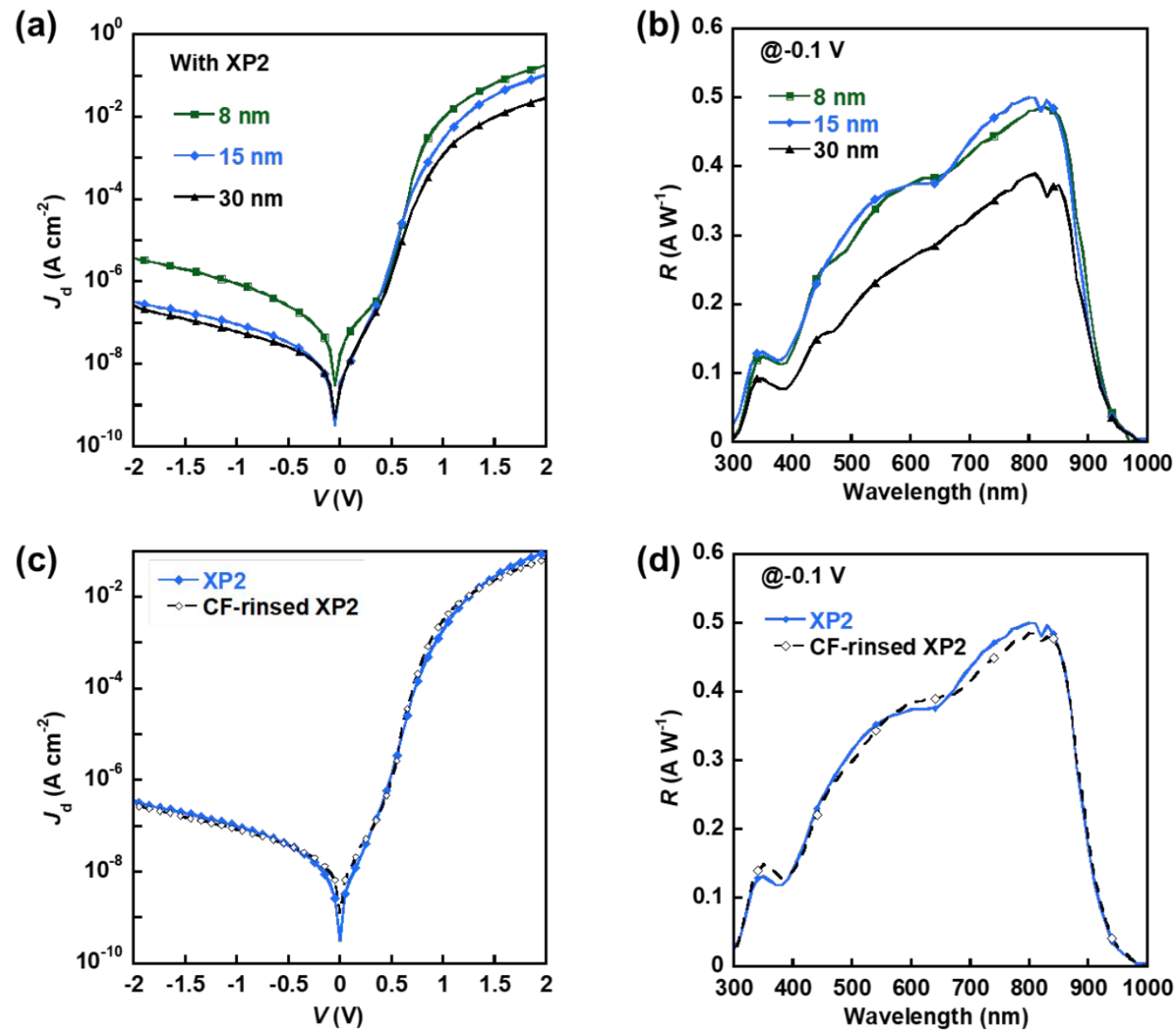

Figure S6. (a) The bias-dependent dark current density and (b) responsivity spectra characteristics of OPDs with XP2 in various thicknesses. (c) The bias-dependent dark current density and (d) responsivity spectra characteristics of OPDs with XP2 rinsed by CF or not. 


\section{Device with Inverted Structure}
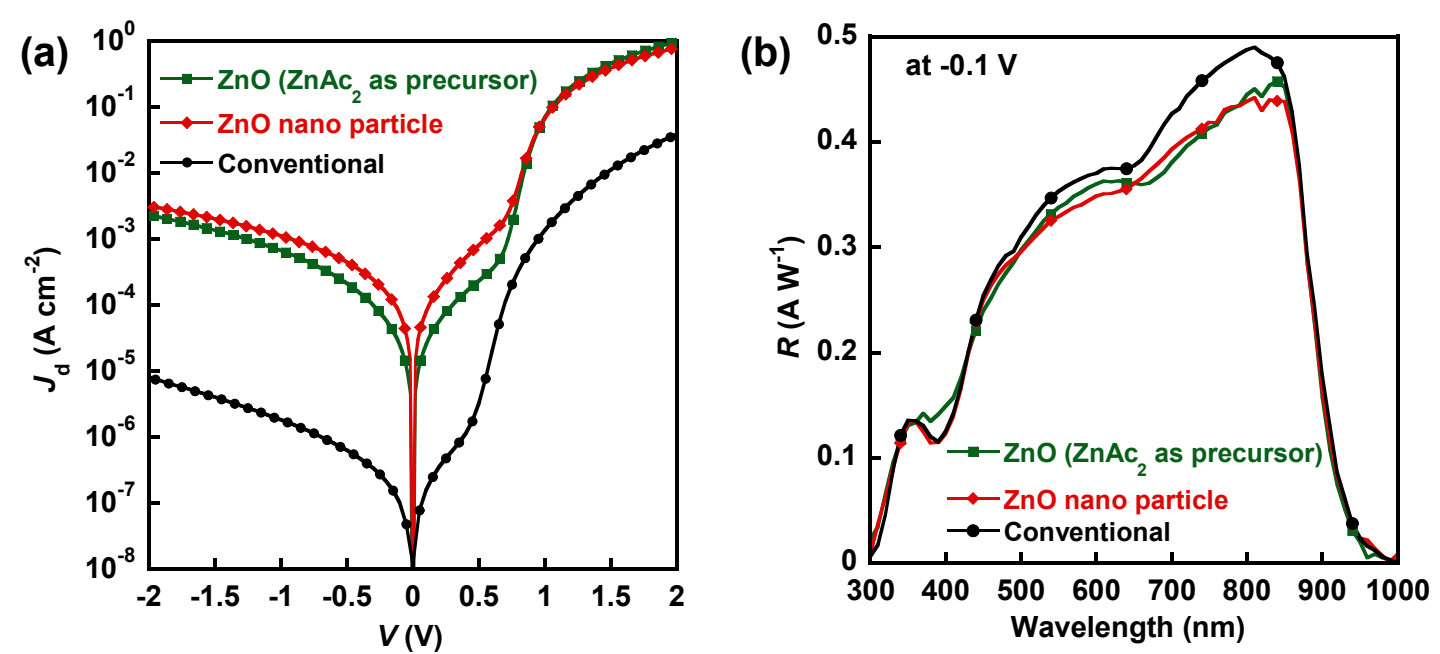

Figure S7. (a) The bias-dependent dark current density and (b) responsivity spectra characteristics of inverted devices and conventional device. The configuration of inverted devices were ITO/ZnO (30 nm)/PFT-OEHp:Y6 $(170 \mathrm{~nm}) / \mathrm{MoO}_{3}(10 \mathrm{~nm}) / \mathrm{Al}$ $(100 \mathrm{~nm})$. The solution-processed $\mathrm{ZnO}$ layer was prepared from $\mathrm{ZnAc}_{2}$ precursor or $\mathrm{ZnO}$ nano particle as article reported elsewhere [S3].

Table S2. The detailed data of inverted and conventional PFT-OEHp:Y6 device.

\begin{tabular}{llll}
\hline & $R_{\max },-0.1 \mathrm{~V}$ & $J_{\mathrm{d}}$ at $-0.1 \mathrm{~V}$ & $D^{*}{ }_{\max },-0.1 \mathrm{~V}$ \\
Device structure & $\left(\mathrm{A} \mathrm{W}^{-1}\right)$ & $\left(\mathrm{A} \mathrm{cm}^{-2}\right)$ & $(\mathrm{Jones})^{a}$ \\
\hline Inverted (sol-gel) & 0.44 & $2.53 \times 10^{-5}$ & $1.56 \times 10^{11}$ \\
Inverted (ZnO NP) & 0.44 & $7.45 \times 10^{-5}$ & $8.98 \times 10^{10}$ \\
Conventional & 0.49 & $1.01 \times 10^{-7}$ & $2.73 \times 10^{12}$ \\
\hline
\end{tabular}

${ }^{a}$ derived from $J_{\mathrm{d}}$. 


\section{Mott-Schottky Analysis}
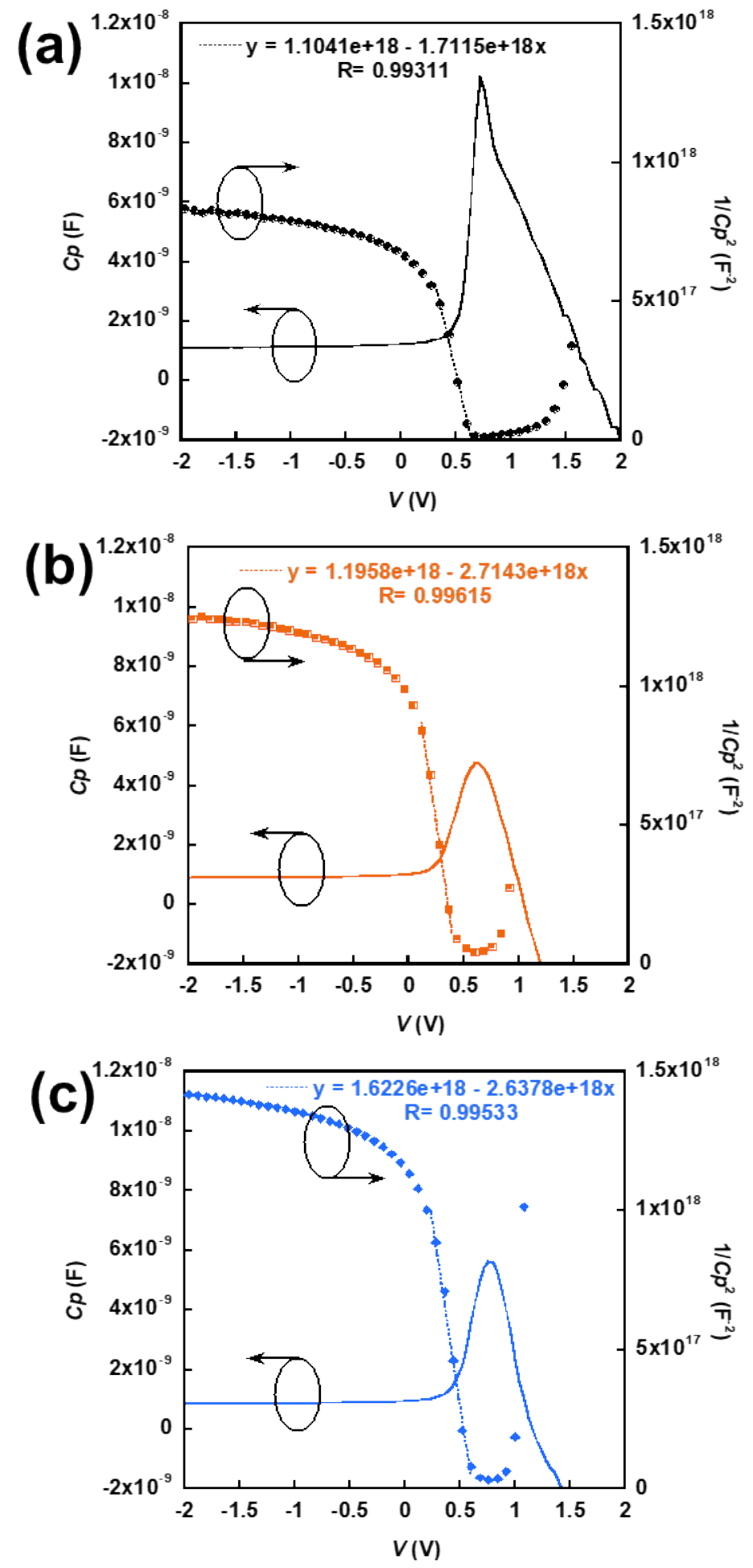

Figure S8. The $C p-V$ and $1 / C p^{2}-V$ characteristics of devices as (a) control or with (b) $\mathrm{XP1}$, (c) XP2 interlayer. The curve fitting for linear regime of $1 / C p^{2}-V$. 


\section{Performance of Peer OPDs}

Table S3. The performance of OPDs containing non-fullerene acceptor.

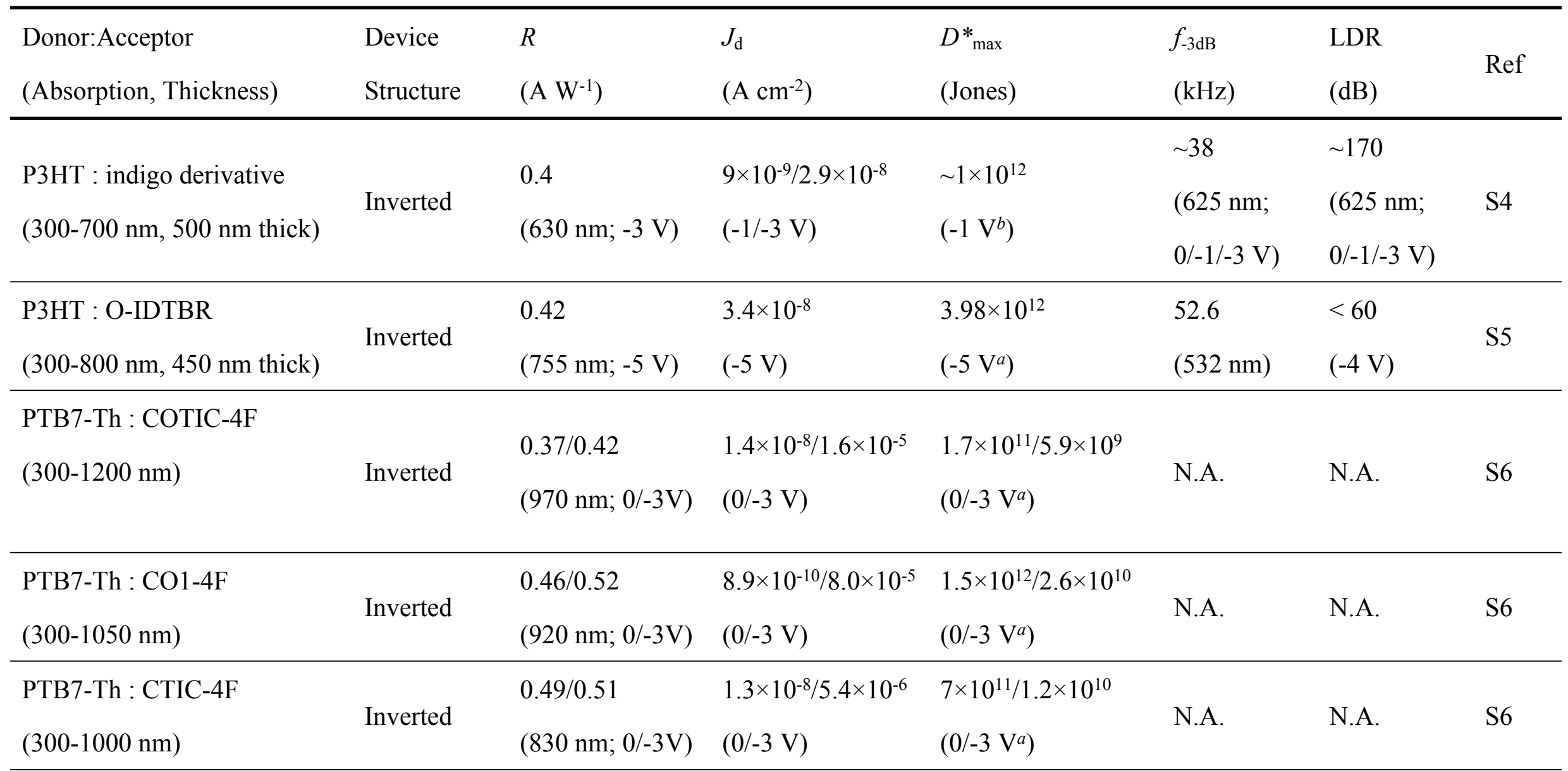




\begin{tabular}{|c|c|c|c|c|c|c|c|}
\hline $\begin{array}{l}\text { PTB7-Th : } \mathrm{CO}_{i} 8 \text { DFIC : } \\
\text { PC }_{71} \mathrm{BM} \\
(300-1000 \mathrm{~nm}, 316 \mathrm{~nm} \text { thick })\end{array}$ & Inverted & $\begin{array}{l}0.35 \\
(670 \mathrm{~nm} ; 0 \mathrm{~V})\end{array}$ & N. A. & $\begin{array}{l}5.6 \times 10^{11} \\
\left(0 \mathrm{~V}^{b}\right)\end{array}$ & $\begin{array}{l}\sim 2000 \\
(528 \mathrm{~nm} ; \\
0 \mathrm{~V})\end{array}$ & $\begin{array}{l}135 \\
(0 \mathrm{~V})\end{array}$ & S7 \\
\hline $\begin{array}{l}\text { PTB7-Th : CO1-4Cl } \\
(300-1100 \mathrm{~nm}, 300 \mathrm{~nm} \text { thick) }\end{array}$ & Inverted & $\begin{array}{l}\sim 0.45 / 0.52 \\
(\sim 950 \mathrm{~nm} \\
-0.1 /-2 \mathrm{~V})\end{array}$ & $\begin{array}{l}5.8 \times 10^{-10} / 7 \times 10^{-9} \\
(-0.1 /-2 \mathrm{~V})\end{array}$ & $\begin{array}{l}3.31 \times 10^{13 />1 \times 10^{13}} \\
\left(-0.1 /-2 V^{a}\right)\end{array}$ & $\begin{array}{l}240 \\
(940 \mathrm{~nm} ; \\
-2 \mathrm{~V})\end{array}$ & $\begin{array}{l}126 \\
(940 \mathrm{~nm}, \\
-0.1 \mathrm{~V})\end{array}$ & S8 \\
\hline $\begin{array}{l}\text { PTB7-Th:IFIC-i-4F:PC }{ }_{71} B M \\
(300-1000 \mathrm{~nm}, 300 \mathrm{~nm} \text { thick) }\end{array}$ & Inverted & $\begin{array}{l}0.38 \\
(\text { peak; } 0 \mathrm{~V})\end{array}$ & $\begin{array}{l}1.23 \times 10^{-11} \\
(0 \mathrm{~V})\end{array}$ & $\begin{array}{l}1.93 \times 10^{14} \\
\left(0 \mathrm{~V}^{a}\right)\end{array}$ & N. A. & $\begin{array}{l}\sim 80 \\
(0 \mathrm{~V})\end{array}$ & S9 \\
\hline $\begin{array}{l}\text { BDT-Th-3T:ITIC } \\
(300-800 \mathrm{~nm}, 300 \mathrm{~nm} \text { thick) }\end{array}$ & Inverted & $\begin{array}{l}\sim 0.31 \\
(625 \mathrm{~nm} ;-0.5 \mathrm{~V})\end{array}$ & $\begin{array}{l}\sim 1 \times 10^{-9} \\
(-0.5 \mathrm{~V})\end{array}$ & $\begin{array}{l}1.4 \times 10^{13} \\
\left(-0.5 \mathrm{~V}^{b}\right)\end{array}$ & $\begin{array}{l}12 \\
(650 \mathrm{~nm} ; \\
-0.5 \mathrm{~V})\end{array}$ & $\begin{array}{l}232 \\
(650 \mathrm{~nm} ; \\
-0.5 \mathrm{~V})\end{array}$ & $\mathrm{S} 10$ \\
\hline
\end{tabular}

${ }^{a}$ derived from dark current density.

${ }^{b}$ derived from noise. 


\section{Reference}

[S1] Zhong, W.; Li, K.; Cui, J.; Gu, T.; Ying, L.; Huang, F.; Cao, Y. Efficient AllPolymer Solar Cells Based on Conjugated Polymer Containing an Alkoxylated ImideFunctionalized Benzotriazole Unit. Macromolecules 2017, 50, 8149-8157.

[S2] Fan, B.; Zhang, D.; Li, M.; Zhong, W.. Zeng, Z.; Ying, L.; Huang, F.; Cao, Y. Achieving over 16\% Efficiency for Single-Junction Organic Solar Cells. Sci. China Chem. 2019, 62, 746-752.

[S3] Sun, Y.; Seo, J. H.; Takacs, C. J.; Seifter, J.; Heeger, A. J. Inverted Polymer Solar Cells Integrated with a Low-Temperature-Annealed Sol-Gel-Derived ZnO Film as an Electron Transport Layer. Adv. Mater. 2011, 23, 1679-1683.

[S4] Kim, I. K.; Li, X.; Ullah, M.; Shaw, P. E.; Wawrzinek, R.; Namdas, E. B.; Lo, S. C., High-Performance, Fullerene-Free Organic Photodiodes Based on a SolutionProcessable Indigo. Adv. Mater. 2015, 27, 6390-6395.

[S5] Gasparini, N.; Gregori, A.; Salvador, M.; Biele, M.; Wadsworth, A.; Tedde, S.; Baran, D.; McCulloch, I.; Brabec, C. J., Visible and Near-Infrared Imaging with Nonfullerene-Based Photodetectors. Adv. Mater. Technol. 2018, 3, 1800104.

[S6] Lee, J.; Ko, S.-J.; Lee, H.; Huang, J.; Zhu, Z.; Seifrid, M.; Vollbrecht, J.; Brus, V. V.; Karki, A.; Wang, H.; Cho, K.; Nguyen, T.-Q.; Bazan, G. C., Side-Chain Engineering of Nonfullerene Acceptors for Near-Infrared Organic Photodetectors and Photovoltaics. ACS Energy Lett. 2019, 4, 1401-1409.

[S7] Li, W.; Xu, Y.; Meng, X.; Xiao, Z.; Li, R.; Jiang, L.; Cui, L.; Zheng, M.; Liu, C.; Ding, L.; Lin, Q., Visible to Near-Infrared Photodetection Based on Ternary Organic Heterojunctions. Adv. Funct. Mater. 2019, 29, 1808948.

[S8] Huang, J.; Lee, J.; Vollbrecht, J.; Brus, V. V.; Dixon, A. L.; Cao, D. X.; Zhu, Z.; Du, Z.; Wang, H.; Cho, K.; Bazan, G. C.; Nguyen, T.-Q., A High-Performance Solution-Processed Organic Photodetector for Near-Infrared Sensing. Adv. Mater. 2020, $32,1906027$.

[S9] Liu, Z.-X.; Lau, T.-K.; Zhou, G.; Li, S.; Ren, J.; Das, S. K.; Xia, R.; Wu, G.; Zhu, 
H.; Lu, X.; Yip, H.-L.; Chen, H.; Li, C.-Z., Achieving Efficient Organic Solar Cells and Broadband Photodetectors via Simple Compositional Tuning of Ternary Blends. Nano Energy 2019, 63, 103807.

[S10] Ko, H.; Park, S.; Son, H. J.; Chung, D. S., Wide-Linear-Dynamic-Range Polymer Photodiode with a New Benzo[1,2-b:4,5-b']dithiophene-Copolymer: The Role of Crystalline Orientation. Chem. Mater. 2020, 32, 3219-3228. 